\title{
Drug Induced Liver Injury: Review with a Focus on Genetic Factors, Tissue Diagnosis, and Treatment Options
}

\author{
Tawfik Khoury ${ }^{\S 1}$, Ayman Abu Rmeileh ${ }^{\S 1}$, Liron Yosha ${ }^{1}$, Ariel A. Benson ${ }^{2}$, Saleh Daher ${ }^{2}$ \\ and Meir Mizrahi ${ }^{\S * 3}$
}

\begin{abstract}
${ }^{1}$ Department of Medicine, Hadassah-Hebrew University Medical Center, Jerusalem, Israel; ${ }^{2}$ Department of Gastroenterology, Hadassah-Hebrew University Medical Center, Jerusalem, Israel; ${ }^{3}$ Center for Advanced Endoscopy, Harvard Medical School, Beth
\end{abstract} Israel Deaconess Medical Center, Boston, MA, USA

\begin{abstract}
Drug-induced liver injury (DILI) is a rare but potentially life threatening adverse drug reaction. DILI may mimic any morphologic characteristic of acute or chronic liver disease, and the histopathologic features of DILI may be indistinguishable from those of other causes of liver injury, such as acute viral hepatitis. In this review article, we provide an update on causative agents, clinical features, pathogenesis, diagnosis modalities, and outcomes of DILI. In addition, we review results of recently reported genetic studies and updates on pharmacological and invasive treatments.

(C) 2015 The Second Affiliated Hospital of Chongqing Medical University. Published by XIA \& HE Publishing Ltd. All rights reserved.
\end{abstract}

\section{Introduction}

Drug-induced liver injury (DILI) is the most frequent indication for drug withdrawal from the pharmaceutical market due to its association with significant adverse effects, morbidity,

\section{Keywords: DILI; Hepatotoxicity; Treatment.}

Abbreviations: ALK, alkaline phosphatase; ALT, alanine aminotransferase; AST, aspartate aminotransferase; BSEP (ABCB11), bile salt export pump; CIOMS, Council for International Organizations of Medical Sciences; CT, computed tomography; CYP-450, cytochrome P450; DDW-J, Digestive Disease Week-Japan; DILI, drug-induced liver injury; DILIN, Drug-Induced Livery Injury Network; DLST, drug lymphocyte stimulation test; FDA, Food and Drug Administration; GSTM1, glutathione s-transferase mu 1; HIV, human immunodeficiency virus; INR, international normalized ratio; MARS, molecular adsorbents recirculatory systems; MDR3 (ABCB4), multidrug resistance P-glycoproteins 3; MELD, model for end-stage liver disease; miRNA, microRNA; MRP2 (ABCC2), multi-drug resistance-associated protein 2; MnSOD, manganese superoxide dismutase; NAC, $\mathrm{N}$-acetylcysteine; NAFLD, non-alcoholic fatty liver disease; NAT2, N-acetyl transferase 2; NSAID, nonsteroidal anti-inflammatory drug; OATP1B1, Organic Anion Transporter Protein B1; PFSA, fractionated plasma separation and adsorption; RCT, randomized control trial; RNA, ribonucleic acid; RUCAM, Roussel Uclaf Causality Assessment Method; SLC, Liver Specific Transporter; SMX, sulfamethoxazole; SNP, single nucleotide polymorphisms; UDCA, ursodeoxycholic acid; UGT, uridine 5'-diphosphoglucuronosyltransferase; UK, United Kingdom; ULN, upper limit of normal; WHO, World Health Organization.

Received: 21 February 2015; Revised: 25 April 2015; Accepted: 28 April 2015 DOI: $10.14218 /$ JCTH.2015.00007

*Correspondence to: Meir Mizrahi, Center for Advanced Endoscopy, Harvard Medical School, Beth Israel Deaconess Medical Center, 330 Brookline Ave, Boston, MA 02215, USA. Tel: +1-617-6672135, Fax: +1-617-6671728, E-mail: mmizrahi@bidmc.harvard.edu

$\S$ These authors contributed equally to this work. and mortality. Several drugs have been removed from the market because of DILI, including bromfenac and troglitazone. ${ }^{1}$ DILI may be divided into intrinsic and idiosyncratic hepatotoxicity, and idiosyncratic injury is further divided into allergic and nonallergic reaction. The intrinsic mechanism is related to dose dependent hepatotoxicity, whereas idiosyncratic hepatotoxicity is not dose dependent and may happen in an unpredictable fashion. Allergic idiosyncratic hepatotoxicity is further characterized by the presence of symptoms and signs typical of an adaptive immune system reaction, including fever, skin reactions, eosinophilia, formation of autoantibodies, and a short latency time particularly after re-exposure.

DILI is responsible for the majority of acute liver failure cases and is now the leading cause for liver transplantation among patients. ${ }^{2}$ Among hospitalized patients with jaundice, $2-10 \%$ of cases are reported to be secondary to liver injury caused by drugs. Furthermore, DILI is responsible for approximately $10 \%$ of all adverse drug reactions in the United States (USA). ${ }^{3}$ Estimates of the incidence of DILI are reported to be as high as $14-24$ cases/100,000 individuals, and estimates show that nearly 44,000 patients per year will develop DILI. ${ }^{4}$

Two prospective surveys of the general population of France and Spain estimated DILI incidence to be 7-14/ 100,000 people, ${ }^{5}$ and retrospective studies from Sweden and the United Kingdom (UK) reported an estimated DILI incidence rate of $2-3 / 100,000$ people per year. ${ }^{6}$ The true incidence of DILI is still largely unknown due to underreporting of adverse drug reactions. Moreover, the presence of confounding factors makes it difficult to determine an accurate DILI incidence. Nonetheless, the World Health Organization (WHO) proclaimed an increasing incidence of DILI since the 1990 s. $^{7}$

\section{Clinical presentation}

The clinical presentation of DILI ranges from asymptomatic abnormal liver function tests to acute and chronic hepatitis. ${ }^{8}$ DILI is classified based on the Council for International Organizations of Medical Sciences (CIOMS), as hepatocellular, cholestatic, or mixed injury. ${ }^{9}$ Approximately $25-30 \%$ of patients who develop DILI display symptoms of an allergic drug reaction, such as fever, rash, and eosinophilia. ${ }^{10}$ Although rare, DILI was implicated in the development of cirrhosis in the long-term follow-up of patients after initial 
presentation of DILI. ${ }^{11}$ Notwithstanding, DILI is often a selflimiting process without chronic consequences, as some patients may need hospitalization, and some may progress to acute liver failure resulting in a high mortality rate. Many drugs can cause DILI, and different drugs have led to varying disease time courses (usually, the resolution of cholestatic liver injury is slower than hepatocellular injury). ${ }^{12}$

Physicians must be aware of time differences in the progression of DILI. For example, trovafloxacin and amoxicillinclavulanic acid can have a lag period of 2-3 weeks between the discontinuation of treatment and the development of idiosyncratic liver injury, as evidenced by elevated liver enzymes. ${ }^{13}$ Depending on several factors, there is wide variability in DILI symptoms, time course, and type of liver injury.

\section{Pathogenesis}

DILI can be mediated by two mechanisms: intrinsic liver injury and idiosyncratic liver injury. Drugs that cause intrinsic liver injury exert hepatotoxic effects either directly by the agent itself or indirectly by its metabolite. In these instances, liver injury is dose dependent. An example of a drug that leads to intrinsic liver injury is acetaminophen. Idiosyncratic hepatotoxicity is the DILI mechanism that develops from the majority of the DILI causing agents. Idiosyncratic liver injury can be caused by metabolic or immunological mechanisms, with the immunological effect resulting from a hypersensitivity reaction. Idiosyncratic liver injury is generally associated with hepatocellular inflammation, while intrinsic liver injury is associated with necrosis and minimal inflammation. Recently, some reports addressed the role of other mechanisms of liver injury, including the role of mitochondrial injury and active drug metabolites in the pathogenesis of DILI. These other mechanisms need further characterization. ${ }^{14}$

The majority of DILI cases are due to idiosyncratic adverse drug reactions, which can involve the liver and other organs, including skin and bone marrow. Five basic theories were developed to explain idiosyncratic drug reactions. These include drug metabolism, the hapten hypothesis, the inflammagen model, the danger hypothesis, and pharmacological interaction. ${ }^{15}$ The large number of theories, different target organs and cells involved in the injury, the diversity of mechanisms involved, and different risk factors limit the development of a simple approach to prevent and predict idiosyncratic DILI. One constant aspect for drugs that cause intrinsic liver injury and drugs with well-documented idiosyncratic DILI is a dose-dependent component. ${ }^{16}$

Although DILI is often related to idiosyncratic reactions, there are other factors that substantially increase the risk of developing idiosyncratic DILI, including genetic susceptibility, older age, environmental factors, female sex, the presence of concomitant diseases, polypharmacy, dosage and duration of treatment, drug formulation, concomitant alcohol consumption, nutritional status (obesity and malnutrition), pre-existing chronic liver disease, and other underlying comorbidities. ${ }^{17}$ Most idiosyncratic drug reactions occur roughly between 1-2 weeks and 2-3 months from the start of drug therapy. ${ }^{18}$ However, there are numerous exceptions describing exposure of a drug for many months prior to DILI, such as in cases of liver injury resulting from nitrofurantoin and ximelagatran. ${ }^{19}$

In many instances, the liver plays an important role in the metabolism and detoxification of drugs, and drugs that are predominantly metabolized by the liver are more frequently associated with hepatic adverse effects than drugs with limited or nonsignificant hepatic metabolism. ${ }^{20}$ The most common drugs in the USA leading to idiosyncratic DILI are antimicrobials, central nervous system drugs, herbal/dietary supplements, and immunomodulatory agents. ${ }^{21}$ However, other drugs, including nonsteroidal anti-inflammatory drugs (NSAIDs) and anticoagulation medications, are associated with idiosyncratic DILI. In an extensive analysis of 461 DILI cases over a 10 year period involving 505 drugs, the antiinfective drugs were found to be most frequently associated with idiosyncratic DILI, with amoxicillin-clavulanate accounting for $12.8 \%$ of these cases. ${ }^{10}$

Despite the complexity of the mechanism underlying DILI, a three step working model has been proposed for its pathogenesis: 1 . drugs, or their metabolites, cause cell stress directly (intrinsic pathway); 2. Immune reactions are triggered; 3. Apoptotic or necrotic cell death, depending on the availability of adenosine triphosphate, is initiated. ${ }^{22}$ Despite this model, the exploration of the mechanism of DILI remains challenging and is at the forefront of research in the field of hepatotoxicity.

\section{Factors associated with increased susceptibility to DILI}

\section{1- Genetic susceptibility}

The occurrence of DILI is associated with genetic vulnerability, and multiple studies have focused on the exploration of single nucleotide polymorphisms (SNP) within transporter genes. ${ }^{4}$ A small study series linked polymorphisms in CYP2C9 and CYP2C19 to DILI. ${ }^{23}$ However, a large Spanish study on DILI patients showed that polymorphisms in these isoforms were not associated with increased susceptibility to DILI, and, based on those findings, genetic variation in CYP2C9 and CYP2C19 may not be considered a DILI risk factor. ${ }^{24}$

CYP2D6, another gene with allelic variants and encoding enzymes with variable degrees of activity, is associated with the development of hepatotoxicity after use of certain pharmaceutical agents, including antidepressants and herbal products. ${ }^{25}$

A potential link was also found between the CYP2E1 genotype and risk for developing DILI. Specifically, the wildtype CYP2E1 genotype is associated with increased susceptibility to DILI, specifically when antituberculosis medications are administered. ${ }^{26}$ However, other studies did not demonstrate this association. ${ }^{27}$ Finally, polymorphic variants of the CYP2B6 gene are associated with ticlopidine-induced liver injury in association with the HLA-A*3303 haplotype in Japanese patients. ${ }^{28}$

Uridine $5^{\prime}$-diphospho-glucuronosyltransferase (UGT) has an important role in metabolism and is linked to the development of DILI. UGT catalyzes glucuronidation reactions, which aid in the detoxification of drugs and endogenous compounds, such as bilirubin and bile acids. Many isoforms of UGT are known, and they are encoded by 19 different genes in humans. ${ }^{29}$ UGT1A1 is involved in bilirubin metabolism, and mutations found in the UGT1A1 gene are responsible for Gilbert's syndrome and hyperbilirubinemia. Drugs that are metabolized by the enzyme expressed by UGT1A1, such as irinotecan, estradiol, and buprenorphine or drugs that inhibit its function, such as indinavir and ketoconazole, have an increased risk for hepatotoxicity in patients with a mutant 
Khoury T. et al: Drug induced liver injury, cause, diagnosis and treatment

UGT1A1 genotype. ${ }^{30}$ Another polymorphic isoform of UGT, UGTB7, is associated with DILI induced by diclofenac by increasing glucuronidation activity. ${ }^{31}$

Mutations in GST mu 1 (GSTM1) and GST theta 1 (GSTT1), other hepatocyte enzymes involved in metabolism and detoxification, may lead to partial deletion, absence of enzymatic activity, and predisposition to DILI. ${ }^{32}$

In addition, MDR3 (ABCB4), MRP2 (ABCC2), and BSEP $(A B C B 11)$ are $A B C$ transporters located at the canalicular hepatocyte membrane and are associated with DILI. Mutations in ABCB4 were described in a patient with hepatocellular DILI following the administration of amoxicillin-clavulanic acid and in a patient with cholestatic DILI after taking risperidone. ${ }^{33}$ Mixed type of DILI associated with mutations in $A B C C 2$ were seen in Korean patients and a mutation of $A B C B 11$ was linked with both drug-induced hepatocellular and drug-induced cholestatic liver injury. ${ }^{34}$

The SLC transporter, OATP1B1, responsible for the influx of many drugs, was shown to have a connection to hepatotoxicity after administration of specific pharmaceuticals, including troglitazone, rifampin, bosentan, methotrexate, and statins. ${ }^{35}$ Polymorphisms in the SLCO1B1 gene were described in cases of drug hypersensitivity. For instance, several SNPs identified in this gene resulted in altered function, which affected levels of statins, methotrexate, and irinotecan and may lead to severe drug toxicities. ${ }^{36}$

Allelic variations of manganese superoxide dismutase (MnSOD), which is involved in the defense against cellular oxidative stress, were shown to possibly increase the susceptibility for DILI, particularly with antituberculosis drugs. ${ }^{37}$ These variations increased the risk for developing cholestatic and mixed types of DILI from drugs and their metabolites in Spanish patients. ${ }^{38}$ Increased levels of MnSOD are also thought to cause accumulation of hydrogen peroxide, which may lead to the occurrence of DILI.

Although many polymorphisms have been identified within CYP450, there is no strong evidence demonstrating a correlation between specific CYP genotypes and DILI. Only a few polymorphisms are associated with hepatotoxicity. Another example of genetic variation leading to DILI susceptibility is mutations in NAT2 (N-acetyl transferase 2), in which some variants were identified as slow or rapid acetylators. Isoniazid, an antituberculosis drug, is initially metabolized by NAT2 prior to oxidation by CYP2E1. Although some felt that rapid acetylators caused isoniazid-induced liver necrosis in the past, ${ }^{39}$ subsequent studies have indicated a strong correlation between slow acetylators and hepatotoxicity caused by antituberculosis drugs. ${ }^{40}$ While genetic factors have been connected to DILI, further research is needed to delineate the exact role that specific genetic mechanisms play in DILI.

\section{2- Adaptive immunological mechanisms}

The adaptive immune system is thought to be involved mechanistically in idiosyncratic DILI, as shown by the presence of immune cells in liver biopsies of DILI patients and by the association of DILI with allergic symptoms. It is hypothesized that either the drug metabolite or parent drug binds to hepatic proteins to form a neoantigen and activates the immune system to generate antibodies against the hepatic proteins. ${ }^{41}$ This mechanism was established with diclofenacinduced liver injury. ${ }^{42}$ Furthermore, autoantibodies against the CYP450 enzymes were reported in cases of DILI; these include ingestion of halothane, tienilic acid, dihydralazine, and anticonvulsant drugs. ${ }^{43}$

Another mechanism thought to involve the immune system in the pathogenesis of DILI is the p-I concept, which suggests that the drug itself directly stimulates T cells. ${ }^{44}$ The p-I mechanism was originally proposed for sulfamethoxazole. $^{45}$ Despite evidence for a role of the immune system in the pathogenesis of DILI, its function in the progression of DILI is unclear because of the natural liver tolerance to antigens from ineffective stimulation of T cells by hepatic antigen presenting cells. ${ }^{46}$

\section{3- Drug-drug interaction and hepatic metabolism}

The risk of idiosyncratic DILI is generally increased with higher doses of a medication and a dose of more than $50 \mathrm{mg}$ daily, especially if the drug is known to have extensive hepatic metabolism. This increased risk is attributed to the generation of reactive intermediaries and subsequent metabolic idiosyncrasy. Furthermore, oral drugs that undergo hepatic metabolism of more than $50 \%$ are associated with increased liver adverse effects, greater incidence of DILI, and increased morbidity and mortality as compared to medications that undergo less than $50 \%$ hepatic metabolism. ${ }^{20}$

\section{4- Underlying disease}

Liver disease is an important factor in the development of both intrinsic and idiosyncratic DILI, as abnormal liver function alters the expression of drug transporter proteins, not only in the liver but also in other organs. ${ }^{47}$ Moreover, underlying liver disease can change the pharmacokinetics of drugs and lead to unexpected effects. It is difficult to predict the action of drugs in patients with liver diseases and, hence, follow-up of serum drug levels and dose adaptations should be performed carefully. ${ }^{48}$

The outcomes and risk for DILI in patients with known chronic liver disease and in whom potential hepatotoxic drugs are used were reviewed previously. ${ }^{49}$ The presence of preexisting liver disease does not definitively increase the susceptibility to DILI, and most drugs can be used safely in these patients. ${ }^{50}$ Still, it is important to remember that a diseased liver with reduced functional capacity and diminished reserve, limits the liver's ability to recover and could worsen the consequences of DILI. ${ }^{51}$ Finally, nonalcoholic fatty liver disease (NAFLD) in both rats and humans was shown to lead to mitochondrial dysfunction, which can significantly increase the risk of DILI. ${ }^{52}$

\section{Role of hepatitis viruses}

Viral hepatitis predisposes patients to the development of DILI in certain situations. Nader et al. reported an increased risk for DILI in hospitalized patients positive for hepatitis C virus receiving treatment with rifampicin, isoniazide, and pyrazinamide for tuberculosis $(8.8 \%$ of the 534 patients developed DILI). ${ }^{53}$ Furthermore, hepatitis $C$ virus infection is a risk factor for increased severity of acetaminophen-induced acute liver injury. ${ }^{54}$ Another study showed that the presence of hepatitis $B$ virus surface antigen was a moderate predictor of DILI but with low precision. ${ }^{55}$ Conversely, a third study concluded that viral hepatitis does not appear to be an established risk factor for INH hepatotoxicity, except in those who are injection drug users. ${ }^{56}$ Patients treated with antiretrovirals for 
human immunodeficiency virus (HIV) infection have a higher risk of severe hepatotoxicity when coinfected with the hepatitis $B$ or $C$ viruses, particularly if the regimen is based on protease inhibitors. ${ }^{57}$ Nonetheless, there is no increase in DILI occurrence in those treated with other antiviral agents. Thus, the presence of viral hepatitis is not a well-established risk factor for the occurrence of DILI.

\section{Classification of liver injury}

The clinical spectrum of liver injury in DILI is classified according to the Council for International Organizations of Medical Sciences (CIOMS). ${ }^{58}$ Hepatocellular DILI is established once there is an isolated elevation of alanine aminotransferase (ALT) greater than two times the upper limit of normal, or when the ratio between ALT to alkaline phosphatase (ALK) is more than five. However, if the ratio of ALT to ALK is below two, the liver injury is classified as cholestatic DILI. A ratio of ALT to ALK between two and five is classified as mixed liver injury.

\section{Diagnosis}

The absence of specific signs and symptoms and the lack of specific criteria and testing make the diagnosis of DILI difficult. The diagnosis of DILI is often one of exclusion (Fig. 1). The manifestations of drug hepatotoxic effects are highly variable, ranging from asymptomatic liver enzymes abnormality to fulminant hepatic failure. Thus, diagnosis of DILI must be based on a comprehensive clinical assessment, including the temporal association between illness and administration of a causative drug. A pattern of liver injury, which is characteristic of the drug, is also helpful for diagnosis as well as exclusion of other causes of acute liver injury.

One of the most difficult issues in the diagnosis of DILI is the determination of causality. There are three main approaches to assess causality. ${ }^{59}$ The gold standard approach is a positive rechallenge test, which is similar to Koch's postulates. However, this approach is not pursued, as it is clinically dangerous. The second method, the ad hoc approach, is also not commonly used. The third, and most widely used, approach is the Roussel Uclaf Causality Assessment Method (RUCAM) scale. This method, demonstrated $86 \%$ sensitivity, $89 \%$ specificity, and positive and negative predictive values of $93 \%$ and $78 \%$, respectively. ${ }^{60}$ RUCAM is a semiquantitative scale, and it contains seven domains, including: onset and ending of the injury after initiation or discontinuation of the suspected agent; progression and course of the reaction; risk factors; concomitant medications; causes of liver injury other than drugs; previous information on the medication; and

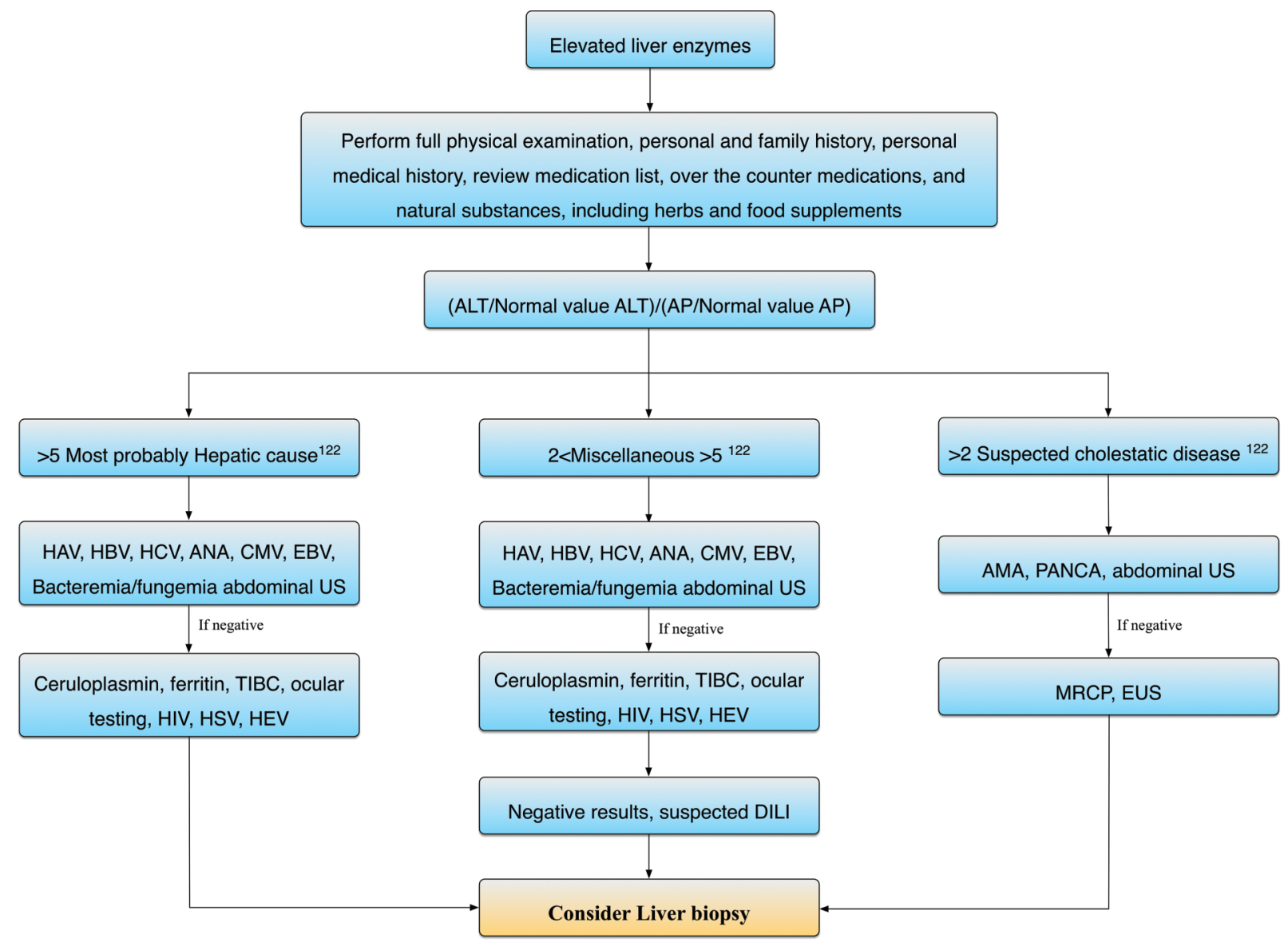

Fig. 1. Algorithm for drug induced liver injury (DILI) diagnosis. ALT, alanine aminotransferase; ALP, alkaline phosphatase; CMV, cytomegalovirus; EBV, Epstein-Barr virus; HCV, hepatitis C virus; HBV, hepatitis B virus, HEV, hepatitis E virus, HSV, herpes simplex virus; MRCP, magnetic resonance cholangiopancreatography; EUS, endoscopic ultrasound; TIBC, total iron binding capacity; HIV, human immunedeficiency virus. 
Khoury T. et al: Drug induced liver injury, cause, diagnosis and treatment

response to readministration, if any. This score assesses causality through assigning a score (ranging between -3 and +3 points) to each domain. The overall score ranges from -5 to +14 . A score greater than eight for an implicated drug suggests a highly probable relationship between the drug and liver injury. Other scores include probable (6-8), possible $(3-5)$, unlikely $(1-2)$, or excluded $(<0) .60$

Maria and Victorino (M\&V) developed another DILI scoring system in 1997 . This system, which is a more simplified scoring system, still uses some components of RUCAM and is referred to as the Clinical Diagnostic Scale or the M\&V scale. The overall score includes five levels: definite, probable, possible, unlikely, and excluded. The M\&V scale is not commonly used because of limitations, particularly the classification of 'definite' only in cases of positive rechallenge and the presence of hypersensitivity features. ${ }^{10}$ Recently, a third diagnostic scoring has been developed in Japan, the Digestive Disease Week-Japan (DDW-J) scale. This scale was also derived from the RUCAM scale, but the DDW-J scale is more accurate than the RUCAM and M\&V scales in the diagnosis DILI. ${ }^{61}$ This scale includes important criteria, such as the in vitro drug lymphocyte stimulation test (DLST). Nonetheless, due to the DLST and lack of standardization, this scale is not frequently used outside of Japan. Further evaluation of the DLST and other lymphocyte-based tests in relation to DILI is needed because of the proposed role of the adaptive immune system in the pathogenesis of DILI. ${ }^{62}$

Despite the development of scoring systems and scales in the evaluation of DILI, DILI is still a diagnosis of exclusion. The definite diagnosis of DILI is supported by the exclusion of other causes of liver injury, such as autoimmunity and viral hepatitis. ${ }^{63}$ Recently, an international DILI expert team reviewed a diagnostic system, and a consensus was established regarding the threshold for defining DILI. The team assigned standardization criteria for the pattern of liver injury severity, chronicity, and assessment of causality. Furthermore, the consensus set an approach to define DILI in the setting of chronic liver disease. ${ }^{64}$ In addition, the Food and Drug Administration (FDA) proposed guidelines for prediction of severe serious DILI as follows: ALT greater than eight times the upper limit of normal, ALT greater than five times the upper limit of normal for 2 weeks, ALT greater than three times the upper limit of normal in association with serum bilirubin greater than two times the upper limit of normal, more than $1.5 \times$ prothrombin time-international normalized ratio (INR), or symptoms of liver injury. In these cases, the guidelines predict severe hepatotoxicity and recommend discontinuation of the culprit drug. ${ }^{65}$

\section{Liver histology in the diagnosis of DILI}

The role of a liver biopsy in the diagnostic work-up of DILI is controversial since there are no unique histological findings of DILI on liver histology. The histological features of DILI can mimic any form of liver injury that is not DILI induced. ${ }^{66}$ Although some histological features, such as abundance of eosinophils and neutrophils, granulomatous hepatitis, and hepatocyte necrosis in the perivenular location, and cholestasis with hepatitis might suggest DILI, overlap exists with many other acute and chronic liver diseases; and no pathognomonic features confirm the diagnosis of DILI. ${ }^{67}$ The absence of portal inflammation with the presence of necrosis in the centrilobular area is relatively characteristic of DILI, but this can still be seen histologically in acute onset autoimmune hepatitis. Currently, no reports address the importance of liver biopsy in causality assessment, but liver histology can be considered compatible with DILI. ${ }^{21}$ In early studies of DILI, histology was considered to be important. However, the advances in methodologies to rule out potential etiologies, such as viral infections, autoimmune diseases, hemochromatosis, and Wilson's disease, and better imaging techniques have decreased the need for a biopsy. Furthermore, if the patient demonstrates a rapid improvement in liver tests following cessation of drug therapy, a routine liver biopsy is not indicated. ${ }^{68}$

Liver biopsy may be helpful when underlying liver disease and/or autoimmune hepatitis is suspected despite negative serological biomarkers. Biopsy can also be used if the suspected agent causing DILI has not been reported previously to cause liver injury or in cases where there is very slow regression or other chronic liver disease. Furthermore, liver biopsy can reveal histological characteristics specific for certain drugs, e.g. steatohepatitis with amiodarone or valproate $^{69}$ or nodular regenerative hyperplasia with azathioprine and 6-thioguanine. ${ }^{70}$ However, the impact of knowing unusual histological patterns on management is uncertain, and there is limited data on the impact of histology on clinical outcomes in DILI. Moreover, studies have shown that there are some histological features that may be present uniformly in DILI cases, such as the presence of portal tract expansion by infiltration of mononuclear cells and eosinophills, centrizonal cholestasis, and focal necrosis. ${ }^{71}$ Ramachandran et al. reported a variety of specific histological patterns induced by various medications. ${ }^{72}$ Recently, a review of liver histopathology in 249 patients in the Drug-Induced Liver Injury Network (DILIN) indicated that five liver injury patterns were responsible for $83 \%$ of the cases. ${ }^{73}$ Poorer outcomes were associated with higher degrees of necrosis, microvesicular steatosis, and a ductular involvement, whereas the presence of intrahepatic eosinophils and/or granulomas was more consistent with a better prognosis. The prognostic use of these histological features is consistent with prior reports. The role of routine liver biopsy in the diagnosis of DILI is still unclear, and in most instances it is not recommended during the early stages of diagnosis. Liver biopsy is certainly indicated in cases where there is no improvement of liver function or when a diagnosis other than DILI is suspected. Additional prospective studies are needed to delineate the role of liver histology in defining and diagnosing DILI.

\section{The role of new serum biomarkers in DILI}

Ongoing research efforts seek to identify new biomarkers for DILI; several proposed biomarker include serum liver injury markers (glutathione S-transferase a, sorbitol dehydrogenase $(\mathrm{SDH})$ ) and hepatocyte mitochondrial dysfunction marker (glutamate dehydrogenase). Moreover, the discovery of circulating serum microRNAs has shown novel tissue specificity of miR-122 and miR-192 for liver injury. Furthermore, serum full-length keratin-18 (K-18) and high mobility group box protein 1 (HMGB-1) have been shown to be a sensitive biomarker for necrosis and cell death, but neither of them is liver specific. $M-30$ is a serum protein that selectively recognizes caspase cleaved neoepitopes of $\mathrm{K}-18$ released from hepatocytes undergoing apoptotic death, while serum $M-65$ reflects total hepatocyte death (apoptosis and necrosis). ${ }^{74,75}$ Recently, the combination of serum M-30 levels with other laboratory parameters was shown to be 
superior to the King's College criteria and Model for EndStage Liver Disease (MELD) score in predicting spontaneous survival in acute liver failure. ${ }^{76}$ Furthermore, DILI secondary to acetaminophen overdose was shown to elevate levels of serum biomarkers of $\mathrm{SDH}$, glutamate dehydrogenase, and HMGB-1. ${ }^{77}$ A panel of these early biomarkers for DILI was recently tested in patients presenting to the hospital after an acetaminophen overdose with initially normal serum liver enzymes levels. ${ }^{78}$ Poorer outcome in acetaminophen overdose was associated with elevated level of acetylated HMGB-1. ${ }^{79}$ Several studies have shown that detection of acetaminophen protein adducts in serum can confirm a diagnosis of acetaminophen hepatotoxicity.

\section{The role serum proteomics in DILI}

Currently, the proteins or pathways involved in the pathogenesis of DILI can be further studied with advanced bioinformatics software. The serum proteomic characteristics of 74 patients in DILIN who had a serum sample collected 2 weeks before the onset of DILI compared to 40 healthy controls were analyzed. ${ }^{80}$ Several proteins were highly elevated in subjects with hepatocellular versus cholestatic DILI, including fructose-bisphosphate aldolase $\mathrm{B}$. This protein was correlated with serum aminotransferases levels at baseline and returned to normal during follow-up. Interestingly, autoantibodies to this protein have previously been reported in patients with troglitazone hepatotoxicity. ${ }^{81}$ Moreover, elevated levels of apolipoprotein $\mathrm{E}$, which is a lipoprotein contained in chylomicrons rich triglyceride, was the major proteome used in distinguishing patients with DILI from controls. A proteomics platform in combination with metabolomics was recently used to distinguish patients who developed ximelagatran hepatotoxicity from unaffected controls. ${ }^{82}$ This data suggested that proteomics might aid in the diagnosis of DILI and guide exploration of causative agents involved in DILI. Thus, further proteomic studies are indicated in this field.

\section{The role of serum cytokines, chemokines and miRNAs in DILI}

Serum chemokines and cytokine levels may also be useful biomarkers for diagnosis and prognosis of DILI. ${ }^{83}$ Recently, the DILIN measured 27 immune analytes in 78 subjects within 2 weeks of DILI onset and after 6 months. ${ }^{84}$ This analysis identified decreases in interleukin- 9 and interleukin-17 and platelet-derived growth factor serum albumin as predictors of early death, suggesting a role for these serum biomarkers in the pathogenesis of DILI. These data are consistent with recent studies showing a role of the Th17 adaptive immunity pathway in the pathogenesis of idiosyncratic DILI. ${ }^{85}$ However, there was no difference in the serum level of interleukin-17 in patients with acute liver failure due to idiosyncratic DILI and those with acetaminophen overdose. ${ }^{86}$

MicroRNAs (miRNAs) are small noncoding RNA molecules (about 22 nucleotides in length) found in plant, animals, and some viruses that function in RNA silencing and posttranscriptional regulation of gene expression. They can be detected in microvesicles in the serum. Although a number of miRNAs are widely expressed, certain miRNAs appear to be tissue specific. miRNAs specifically expressed in the liver include miR-122, miR-21, and miR-192. Liver-derived miRNAs may represent specific biomarkers of acetaminophen-induced DILI. However, early in the course of livery injury, these miRNAs are elevated. ${ }^{87}$ In addition, they appear to have a prognostic role when considering liver transplant in acetaminophen hepatotoxicity. ${ }^{88}$ Given their liver tissue specificity, assay quantification availability, and short half-life, these miRNAs are important biomarkers for severe acute liver injury.

\section{Pharmacogenomics into the pathogenesis of DILI}

Human leukocyte antigen (HLA) is the locus for genes that encode highly polymorphic proteins on the surface of the cells that are responsible for regulating the immune system in humans. A growing number of other immunological reactions, including dermatologic reactions and idiosyncratic DILI, have also been associated with various HLA alleles. ${ }^{89}$ In most instances, there are a series of drug-protein modifications that produce an intermediate or reactive metabolite that is essential for forming the immunogenic hapten. Hapten then binds the antigen binding location on HLA. Recently, different HLA susceptibility alleles to carbamazepine hypersensitivity in Chinese and European subjects were reported. ${ }^{90}$ Since HLA polymorphisms are restricted to each ethnicity, the absence of a particular genetic susceptibility to DILI in one patient population ethnicity does not necessarily exclude a positive association in another group population.

\section{Treatment}

DILI has a wide range of manifestations, from silent biochemical abnormalities to severe fulminant hepatitis with jaundice. Liver injury secondary to DILI often improves following discontinuation of the suspected offending drug. There also may be spontaneous regression of liver injury without discontinuation of the causative drug. Therefore, the discontinuation of a drug should be carefully evaluated, in consideration of the significance of the medication and the degree of damage being caused by its administration. ${ }^{91}$ The association of transaminase elevation in combination with clinical jaundice, secondary to the administration of drugs, was associated with serious liver injury and poor prognosis (fatality rate of $10 \%$ for many drugs). ${ }^{92}$ Due to those findings of increased mortality, Hy's law for monitoring and estimating the severity of DILI was developed. HY's law includes elevation of liver enzymes (aspartate aminotransferase (AST) or ALT more than 3 times upper limit of normal (ULN) or ALP more than 1.5 times ULN) in combination with elevated bilirubin (more than 3 times ULN) at any time after starting a new drug. If HY's law is met, it predicts severe liver injury; and termination of drug use is recommended. However, two recent studies have shown that the mortality rate is high in patients with DILI and jaundice even after the discontinuation of the suspected agents. ${ }^{10}$ The predicted mortality in Hy's law has been confirmed by several international studies in Sweden, Spain, and the USA, which demonstrated a $9-12 \%$ rate of mortality or liver transplantation in patients who developed severe DILI with jaundice. ${ }^{93}$ Once DILI is suspected or identified, management begins with the prompt discontinuation of the suspected agents along with supportive measures and monitoring. There are no reports of beneficial therapies, other than the use of $\mathrm{N}$-acetylcysteine for acetaminophen-induced liver injury. ${ }^{94}$ Empiric use of corticosteroids in acute liver failure due to DILI is not recommended due to the lack of benefit in previously reported studies but is still sometimes used in patients with severe DILI. ${ }^{95}$ Steroid 
Khoury T. et al: Drug induced liver injury, cause, diagnosis and treatment

therapy may be useful in DILI cases associated with hypersensitivity features, such as is the case with carbamazepineinduced hepatotoxicity. ${ }^{95}$ Furthermore, steroids also exert beneficial effects in the treatment of drug-induced autoimmune hepatitis, such as nitrofurantoin and minocycline. ${ }^{96}$

In two acute liver failure trials, which included 104 patients and 12 patients with DILI, steroids failed to demonstrate any benefit. Moreover, in those trials, patients in the steroid treatment group tended to have a worse prognosis. ${ }^{97} \mathrm{~A}$ recent retrospective study of 300 patients who were hospitalized with DILI showed that combination treatment with ursodeoxycholic acid (UDCA) and oral steroids was beneficial for nonacetaminophen DILI with acute liver failure. The combination therapy had a significantly positive effect on resolution and recovery time and prognosis. ${ }^{98}$

Antioxidants have been used for the treatment of severe DILI, and, specifically, N-acetylcysteine (NAC) is considered the treatment of choice for acetaminophen-induced liver injury. ${ }^{99}$ The benefits of NAC in nonacetaminophen DILI has still yet to be elucidated. In one trial that included 173 patients with nonacetaminophen liver injury, the administration of continuous intravenous NAC over 3 days was not associated with improved survival. However, a subset of patients with early stage mild acute liver injury did show an increased rate of spontaneous improvement and better prognosis. ${ }^{100}$ In another study, intravenous NAC was beneficial in the treatment of acute liver failure secondary to idiosyncratic DILI other than paracetamol. ${ }^{101}$

Plasma exchange is another therapeutic modality that had been used for the treatment of acute liver failure. The mechanism of this modality lies in the removal of toxins that accumulate in hepatic failure and the addition of coagulation factors to correct the coagulopathy from liver injury. ${ }^{102}$ Multiple studies have shown the efficacy of plasma exchange in reducing the level of nitrogenous waste products (ammonia and urea), improving coagulopathy, and improving encephalopathy in patients with acute liver failure. ${ }^{103}$ Other studies have demonstrated that plasma exchange exerts beneficial effects on survival in acute liver failure secondary to acetaminophen DILI and in patients with residual liver functional capacity. ${ }^{104}$ In acute liver failure, other benefits stem from the removal of inflammatory cytokines and effects on regulatory T cells. ${ }^{105}$ Therefore, plasma exchange exerts positive prognostic and survival value over symptomatic treatment alone in cases of acute liver failure secondary to DILI. Still, other studies have shown that plasma exchange has only minor survival benefits on patients with pre-existing chronic liver disease. ${ }^{106}$

Molecular adsorbents recirculatory systems (MARS) and fractionated plasma separation and adsorption (PFSA) are advanced treatment options in cases of hepatic failure, although they have no beneficial survival effect in randomized control trials (RCTs) of patients with acute and chronic liver failure. ${ }^{107,108}$ Even though plasma exchange may be superior to MARS and PFSA in removing cytokines, MARS and PFSA have decreased risk of infections and allergic reactions. ${ }^{109}$ Thus, plasma exchange may be preferable for the support of fulminant hepatic failure due to its capacity to remove inflammatory cytokines and protein bound toxic molecules, specifically in patients with acute liver failure without pre-existing chronic liver disease. Although treatment options in DILI are limited, UDCA does have a positive effect on DILI, particularly in cases of cholestatic injury, by protecting hepatocytes from the toxic effects of bile acids. ${ }^{110}$
In order to provide adequate supportive care and management in acute fulminant hepatic failure (including from DILI), patients should be cared for in the setting of an intensive care unit in a liver transplant center. Once acute liver failure develops, liver transplantation may be the sole treatment option. ${ }^{11}$

\section{The course and the natural history of DILI after the acute episode: does DILI lead to chronic liver disease?}

The progression of acute liver injury from DILI to chronic liver disease and liver cirrhosis has been reported with different drugs. ${ }^{112}$ A prospective study on Spanish patients revealed the development of chronic liver injury in $6 \%$ of patients followed for a period of 20 months after initial DILI. In this study, chronic liver injury was defined by the presence of abnormal liver function tests for more than 3 months after DILI onset. Drugs most commonly associated with chronic liver disease were amoxicillin/clavulanate, bentazepam, and atorvastatin. Patients with a cholestatic liver injury pattern were more susceptible to progress to chronic liver injury. ${ }^{113}$ Similar results were shown among Swedish patients, as only three out of 50 (6\%) patients who presented with DILI had persistently abnormal liver tests after a median follow-up of 48 months. ${ }^{114}$

The long term consequences of DILI was further evaluated in a study that followed patients for a period of 10 years, and the progression of DILI to significant liver disease after severe DILI was rare. Twenty-three out of 685 (3\%) DILI patients were hospitalized for liver disease during the study period, and five out of 685 patients had liver-related mortality. Eight patients developed liver cirrhosis, and among those eight patients, five cirrhotic patients did not have other known causes of liver disease other than the past episode of DILI. The patients who developed liver-related morbidity and mortality had longer duration of therapy with the suspected DILI causing agent. ${ }^{11}$

\section{Prognosis}

DILI patients with asymptomatic and mild disease are expected to recover completely. In addition, most patients with symptomatic DILI are expected to recover after discontinuation of the causative drug. With supportive care most patients will not have residual clinical, laboratory, radiological, or histological evidence of liver disease. ${ }^{93} \mathrm{~A}$ favorable prognosis for recovery is expected in the majority of patients with DILI. For example, in one cohort, 712 of 784 (91\%) DILI patients with jaundice recovered. ${ }^{93}$ Conversely, patients with severe DILI associated with acute liver failure and concomitant coagulopathy and encephalopathy had a poor prognosis ${ }^{115}$. Even though the incidence of DILI is more common in males, females tend to develop a more severe course of DILI, leading to acute decompensated liver failure. The prognosis of acetaminophen-induced DILI treated by $\mathrm{N}$-acetylcycsteine is better than the prognosis of DILI from other drugs. Although most of the patients recover from DILI, there are patients who develop chronic liver disease, with the incidence of developing chronic DILI in one Spanish registry reported to be $6 \% .113$

Patients who fulfill Hy's Law and patients with a predominant cholestatic type of DILI have a worse prognosis and increased mortality. ${ }^{10,93}$ The presence of eosinophilic hepatic infiltration with peripheral eosinophilia has a positive prognostic value in DILI, ${ }^{116}$ as demonstrated in a Spanish study 
that showed that eosinophilia was more common in patients who recovered from DILI. In that study, approximately $5 \%$ of the patients who died or underwent liver transplantation had peripheral eosinophilia, while $23 \%$ eosinophilia was seen in patients who recovered. ${ }^{10}$ Once DILI patients develop or present with coagulopathy and encephalopathy, the prognosis is very poor, with approximately $60-80 \%$ mortality in the absence of liver transplantation. ${ }^{117}$ Furthermore, an improvement of liver enzymes is not always predictive of an improved prognosis, as the reduction of serum liver enzymes might herald poor prognostic value due to massive hepatocyte death. ${ }^{118}$

Scoring systems have been developed to assess which patients are candidates for liver transplantation in hepatic disease. The model for end-stage liver disease (MELD) score, which is based on bilirubin, serum creatinine, and INR, can be used to predict the short term mortality rate among acetaminophen-induced liver injury. ${ }^{119}$ A second score, the King's College Hospital Criteria for fulminant hepatic failure in both acetaminophen and nonacetaminophen-induced acute liver failure, is used to identify candidates for liver transplantation. ${ }^{120}$ In addition, recent radiological assessment scores of hepatic volume by computed tomography (CT) can be helpful in predicting prognosis in DILI patients. ${ }^{121}$

\section{Conclusions}

DILI remains one of the most common causes of acute liver failure and is a challenging clinical entity due to the lack of specific diagnostic and prognostic markers. The pathogenesis of DILI is diverse and genetic susceptibility may play a role in the development of DILI, especially idiosyncratic DILI. The clinical spectrum of DILI is complex, and, although DILI is still a diagnosis of exclusion, diagnostic accuracy may be improved by using causality assessment scores. The various histological patterns of DILI reported in studies and the lack of specific histological characteristics of DILI also contribute to the difficulty in diagnosing DILI. Finally, the basis of treatment in DILI is discontinuation of the suspected causative agent and supportive measures. If signs of hypersensitivity are present, then evidence supports the use of steroids. Further RCTs are needed to identify definite biomarkers for the diagnosis of DILI and to explore the role of specific treatments.

\section{Conflict of interest}

None

\section{Author contributions}

Study concept and design (TK, AAR, MM), acquisition of data (TK, AAR, LY, SD, MM), analysis and interpretation of data (TK, $A A R)$, drafting of the manuscript (TK, AAR, LY, AAB, SD, MM), critical revision of the manuscript for important intellectual content (TK, AAR, MM), technical or material support (TK, $M M)$, study supervision (TK, MM), review of the final manuscript (TK, AAR, LY, AAB, SD, MM).

\section{References}

[1] Holt MP, Ju C. Mechanisms of drug-induced liver injury. AAPS J 2006;8: E48-E54. doi:10.1208/aapsj080106.

[2] Lee WM. Acute liver failure in the United States. Semin Liver Dis 2003;23: 217-226. doi:10.1055/s-2003-42641.
[3] Lewis JH. Drug-induced liver disease. Med Clin North Am 2000;84:1275$1311, x$.

[4] Au JS, Navarro VJ, Rossi S. Review article: Drug-induced liver injury-its pathophysiology and evolving diagnostic tools. Aliment Pharmacol Ther 2011;34:11-20. doi:10.1111/j.1365-2036.2011.04674.x.

[5] Sgro C, Clinard F, Ouazir K, Chanay H, Allard C, Guilleminet C, et al. Incidence of drug-induced hepatic injuries: a French population-based study. Hepatology 2002;36:451-455. doi:10.1053/jhep.2002.34857.

[6] De Valle MB, Av Klinteberg V, Alem N, Olsson R, Bjornsson E. Drug-induced liver injury in a Swedish University hospital out-patient hepatology clinic. Aliment Pharmacol Ther 2006;24:1187-1195. doi:10.1111/j.1365-2036. 2006.03117.x.

[7] Bjornsson E, Olsson R. Suspected drug-induced liver fatalities reported to the WHO database. Dig Liver Dis 2006;38:33-38. doi:10.1016/j.dld.2005. 06.004.

[8] Mayoral W, Lewis JH, Zimmerman H. Drug-induced liver disease. Curr Opin Gastroenterol 1999;15:208-216.

[9] Watkins PB, Seeff LB. Drug-induced liver injury: summary of a single topic clinical research conference. Hepatology 2006;43:618-631. doi:10. 1002/hep.21095.

[10] Andrade RJ, Lucena MI, Fernandez MC, Pelaez G, Pachkoria K, Garcia-Ruiz $E$, et al. Drug-induced liver injury: an analysis of 461 incidences submitted to the Spanish registry over a 10 -year period. Gastroenterology $2005 ; 129$ : 512-521. doi:10.1053/j.gastro.2005.05.006.

[11] Bjornsson E, Davidsdottir L. The long-term follow-up after idiosyncratic drug-induced liver injury with jaundice. J Hepatol 2009;50:511-517. doi: 10.1016/j.jhep.2008.10.021.

[12] Lewis JH, Zimmerman HJ. Drug- and chemical-induced cholestasis. Clin Liver Dis 1999;3:433-464, vii.

[13] Lucena MI, Andrade RJ, Rodrigo L, Salmeron J, Alvarez A, Lopez-Garrido MJ, et al. Trovafloxacin-induced acute hepatitis. Clin Infect Dis 2000;30: 400-401. doi:10.1086/313680.

[14] Adams DH, Ju C, Ramaiah SK, Uetrecht J, Jaeschke H. Mechanisms of immune-mediated liver injury. Toxicol Sci 2010;115:307-321. doi: 10. 1093/toxsci/kfq009.

[15] Uetrecht J. Idiosyncratic drug reactions: past, present, and future. Chem Res Toxicol 2008;21:84-92. doi:10.1021/tx700186p.

[16] Lammert C, Einarsson S, Saha C, Niklasson A, Bjornsson E, Chalasani N. Relationship between daily dose of oral medications and idiosyncratic druginduced liver injury: search for signals. Hepatology 2008;47:2003-2009. doi:10.1002/hep.22272.

[17] Andrade RJ, Robles M, Ulzurrun E, Lucena MI. Drug-induced liver injury: insights from genetic studies. Pharmacogenomics 2009;10:1467-1487. doi: $10.2217 /$ pgs.09.111.

[18] Larrey D. Epidemiology and individual susceptibility to adverse drug reactions affecting the liver. Semin Liver Dis 2002;22:145-155. doi:10.1055/s2002-30105.

[19] Lee WM, Larrey D, Olsson R, Lewis JH, Keisu M, Auclert L, et al. Hepatic findings in long-term clinical trials of ximelagatran. Drug Saf 2005;28: 351-370.

[20] Lammert C, Bjornsson E, Niklasson A, Chalasani N. Oral medications with significant hepatic metabolism at higher risk for hepatic adverse events. Hepatology 2010;51:615-620. doi:10.1002/hep.23317.

[21] Chalasani N, Fontana RJ, Bonkovsky HL, Watkins PB, Davern T, Serrano J, et al. Causes, clinical features, and outcomes from a prospective study of drug-induced liver injury in the United States. Gastroenterology 2008;135: 1924-1934,1934.e1-e4. doi:10.1053/j.gastro.2008.09.011.

[22] Russmann S, Kullak-Ublick GA, Grattagliano I. Current concepts of mechanisms in drug-induced hepatotoxicity. Curr Med Chem 2009;16:30413053. doi:10.2174/092986709788803097.

[23] Larrey D, Pageaux GP. Genetic predisposition to drug-induced hepatotoxicity. J Hepatol 1997;26:12-21.

[24] Pachkoria K, Lucena MI, Ruiz-Cabello F, Crespo E, Cabello MR, Andrade RJ. Genetic polymorphisms of CYP2C9 and CYP2C19 are not related to druginduced idiosyncratic liver injury (DILI). Br J Pharmacol 2007;150:808815. doi:10.1038/sj.bjp.0707122.

[25] Maurer HH, Kraemer T, Springer D, Staack RF. Chemistry, pharmacology, toxicology, and hepatic metabolism of designer drugs of the amphetamine (ecstasy), piperazine, and pyrrolidinophenone types: a synopsis. Ther Drug Monit 2004;26:127-131.

[26] Sun F, Chen Y, Xiang Y, Zhan S. Drug-metabolising enzyme polymorphisms and predisposition to anti-tuberculosis drug-induced liver injury: a metaanalysis. Int J Tuberc Lung Dis 2008;12:994-1002.

[27] An HR, Wu XQ, Wang ZY, Zhang JX, Liang Y. NAT2 and CYP2E1 polymorphisms associated with antituberculosis drug-induced hepatotoxicity in Chinese patients. Clin Exp Pharmacol Physiol 2012;39:535-543. doi:10. 1111/j.1440-1681.2012.05713.x.

[28] Ariyoshi N, Iga Y, Hirata K, Sato Y, Miura G, Ishii I, et al. Enhanced susceptibility of HLA-mediated ticlopidine-induced idiosyncratic hepatotoxicity by 
CYP2B6 polymorphism in Japanese. Drug Metab Pharmacokinet 2010;25: 298-306. doi:10.2133/dmpk.25.298.

[29] Miners JO, McKinnon RA, Mackenzie PI. Genetic polymorphisms of UDPglucuronosyltransferases and their functional significance. Toxicology 2002;181-182:453-456. doi:10.1016/S0300-483X(02)00449-3.

[30] Chang JC, Liu EH, Lee CN, Lin YC, Yu MC, Bai KJ, et al. UGT1A1 polymorphisms associated with risk of induced liver disorders by anti-tuberculosis medications. Int J Tuberc Lung Dis 2012;16:376-378. doi:10.5588/ijtld. 11.0404

[31] Daly AK, Aithal GP, Leathart JB, Swainsbury RA, Dang TS, Day CP. Genetic susceptibility to diclofenac-induced hepatotoxicity: contribution of UGT2B7, CYP2C8, and ABCC2 genotypes. Gastroenterology 2007;132:272-281. doi:10.1053/j.gastro.2006.11.023.

[32] Martinez C, Garcia-Martin E, Ladero JM, Herraez O, Ortega L, Taxonera C, et al. GSTT1 and GSTM1 null genotypes may facilitate hepatitis C virus infection becoming chronic. J Infect Dis 2007;195:1320-1323. doi:10. $1086 / 513569$.

[33] Lang $C$, Meier $Y$, Stieger $B$, Beuers $U$, Lang $T$, Kerb $R$, et al. Mutations and polymorphisms in the bile salt export pump and the multidrug resistance protein 3 associated with drug-induced liver injury. Pharmacogenet Genomics 2007; 17:47-60.

[34] Choi JH, Ahn BM, Yi J, Lee JH, Nam SW, Chon CY, et al. MRP2 haplotypes confer differential susceptibility to toxic liver injury. Pharmacogenet Genomics 2007; 17:403-415.

[35] Xiang X, Han Y, Neuvonen M, Pasanen MK, Kalliokoski A, Backman JT, et al. Effect of SLCO1B1 polymorphism on the plasma concentrations of bile acids and bile acid synthesis marker in humans. Pharmacogenet Genomics 2009; 19:447-457. doi:10.1097/FPC.0b013e32832bcf7b.

[36] Konig J, Seithel A, Gradhand U, Fromm MF. Pharmacogenomics of human OATP transporters. Naunyn Schmiedebergs Arch Pharmacol 2006;372: 432-443. doi:10.1007/s00210-006-0040-y.

[37] Huang YS, Su WJ, Huang YH, Chen CY, Chang FY, Lin HC, et al. Genetic polymorphisms of manganese superoxide dismutase, $\mathrm{NAD}(\mathrm{P}) \mathrm{H}$ :quinone oxidoreductase, glutathione S-transferase $\mathrm{M} 1$ and $\mathrm{T} 1$, and the susceptibility to drug-induced liver injury. J Hepatol 2007;47:128-134. doi: 10.1016/j. jhep.2007.02.009.

[38] Lucena MI, Garcia-Martin E, Andrade RJ, Martinez C, Stephens C, Ruiz JD, et al. Mitochondrial superoxide dismutase and glutathione peroxidase in idiosyncratic drug-induced liver injury. Hepatology 2010;52:303-312. doi:10.1002/hep.23668.

[39] Mitchell JR, Zimmerman HJ, Ishak KG, Thorgeirsson UP, Timbrell JA, Snodgrass WR, et al. Isoniazid liver injury: clinical spectrum, pathology, and probable pathogenesis. Ann Intern Med 1976;84:181-192.

[40] Huang YS, Chern HD, Su WJ, Wu JC, Lai SL, Yang SY, et al. Polymorphism of the $\mathrm{N}$-acetyltransferase 2 gene as a susceptibility risk factor for antituberculosis drug-induced hepatitis. Hepatology 2002;35:883-889. doi:10. 1053/jhep.2002.32102.

[41] Park BK, Pirmohamed M, Kitteringham NR. Role of drug disposition in drug hypersensitivity: a chemical, molecular, and clinical perspective. Chem Res Toxicol 1998;11:969-988. doi:10.1021/tx980058f.

[42] Boelsterli UA, Zimmerman HJ, Kretz-Rommel A. Idiosyncratic liver toxicity of nonsteroidal antiinflammatory drugs: molecular mechanisms and pathology. Crit Rev Toxicol 1995;25:207-235. doi:10.3109/10408449509089888.

[43] Dansette PM, Bonierbale E, Minoletti C, Beaune PH, Pessayre D, Mansuy D. Drug-induced immunotoxicity. Eur J Drug Metab Pharmacokinet 1998;23: 443-451.

[44] Pichler WJ. Pharmacological interaction of drugs with antigen-specific immune receptors: the p-i concept. Curr Opin Allergy Clin Immunol 2002; 2:301-305.

[45] Burkhart C, von Greyerz S, Depta JP, Naisbitt DJ, Britschgi M, Park KB, et $\mathrm{al}$. Influence of reduced glutathione on the proliferative response of sulfamethoxazole-specific and sulfamethoxazole-metabolite-specific human CD4+ T-cells. Br J Pharmacol 2001;132:623-630. doi:10.1038/sj. bjp.0703845.

[46] Ju C, Pohl LR. Tolerogenic role of Kupffer cells in immune-mediated adverse drug reactions. Toxicology 2005;209:109-112. doi:10.1016/j.tox.2004. 12.017 .

[47] Ikemura K, Iwamoto T, Okuda M. Altered functions and expressions of drug transporters in liver, kidney and intestine in disorders of local and remote organs: possible role of oxidative stress in the pathogenesis. Expert Opin Drug Metab Toxicol 2009;5:907-920. doi:10.1517/17425250903008525.

[48] Verbeeck RK. Pharmacokinetics and dosage adjustment in patients with hepatic dysfunction. Eur J Clin Pharmacol 2008;64:1147-1161. doi:10. 1007/s00228-008-0553-z.

[49] Gupta NK, Lewis JH. Review article: The use of potentially hepatotoxic drugs in patients with liver disease. Aliment Pharmacol Ther 2008;28: 1021-1041. doi:10.1111/j.1365-2036.2008.03822.x.

[50] Lewis JH. The rational use of potentially hepatotoxic medications in patients with underlying liver disease. Expert Opin Drug Saf 2002;1:159-172.
[51] Amarapurkar DN. Prescribing medications in patients with decompensated liver cirrhosis. Int J Hepatol 2011;2011:519-526. doi:10.4061/2011/ 519526.

[52] Donthamsetty S, Bhave VS, Mitra MS, Latendresse JR, Mehendale HM. Nonalcoholic fatty liver sensitizes rats to carbon tetrachloride hepatotoxicity. Hepatology 2007;45:391-403. doi:10.1002/hep.21530.

[53] Nader LA, de Mattos AA, Picon PD, Bassanesi SL, De Mattos AZ, Pineiro Rodriguez M. Hepatotoxicity due to rifampicin, isoniazid and pyrazinamide in patients with tuberculosis: is anti-HCV a risk factor? Ann Hepatol 2010;9: 70-74.

[54] Nguyen GC, Sam J, Thuluvath PJ. Hepatitis C is a predictor of acute liver injury among hospitalizations for acetaminophen overdose in the United States: a nationwide analysis. Hepatology 2008;48:1336-1341. doi:10. 1002/hep.22536.

[55] de Castro L, do Brasil PE, Monteiro TP, Rolla VC. Can hepatitis B virus infection predict tuberculosis treatment liver toxicity? Development of a preliminary prediction rule. Int J Tuberc Lung Dis 2010;14:332-340.

[56] Bliven EE, Podewils LJ. The role of chronic hepatitis in isoniazid hepatotoxicity during treatment for latent tuberculosis infection. Int J Tuberc Lung Dis 2009;13:1054-1060.

[57] Bruno R, Sacchi P, Maiocchi L, Patruno S, Filice G. Hepatotoxicity and antiretroviral therapy with protease inhibitors: A review. Dig Liver Dis 2006;38: 363-373. doi:10.1016/j.dld.2006.01.020.

[58] Benichou C. Criteria of drug-induced liver disorders. Report of an international consensus meeting. J Hepatol 1990;11:272-276.

[59] Hutchinson TA, Lane DA. Assessing methods for causality assessment of suspected adverse drug reactions. J Clin Epidemiol 1989;42:5-16. doi: 10.1016/0895-4356(89)90020-6.

[60] Benichou C, Danan G, Flahault A. Causality assessment of adverse reactions to drugs-II. An original model for validation of drug causality assessment methods: case reports with positive rechallenge. J Clin Epidemiol 1993;46: 1331-1336. doi:10.1016/0895-4356(93)90102-7.

[61] Takikawa H, Takamori Y, Kumagi T, Onji M, Watanabe M, Shibuya A, et al. Assessment of 287 Japanese cases of drug induced liver injury by the diagnostic scale of the International Consensus Meeting. Hepatol Res 2003;27: 192-195.

[62] Watkins PB. Biomarkers for the diagnosis and management of drug-induced liver injury. Semin Liver Dis 2009;29:393-399. doi:10.1055/s-00291240008.

[63] Davern TJ, Chalasani N, Fontana RJ, Hayashi PH, Protiva P, Kleiner DE, et al. Acute hepatitis $\mathrm{E}$ infection accounts for some cases of suspected druginduced liver injury. Gastroenterology 2011;141:1665-1672.e1-e9. doi: 10.1053/j.gastro.2011.07.051.

[64] Aithal GP, Watkins PB, Andrade RJ, Larrey D, Molokhia M, Takikawa H, et al. Case definition and phenotype standardization in drug-induced liver injury. Clin Pharmacol Ther 2011;89:806-815. doi:10.1038/clpt.2011.58.

[65] Norris W, Paredes AH, Lewis JH. Drug-induced liver injury in 2007. Curr Opin Gastroenterol 2008;24:287-297. doi:10.1097/MOG.0b013e3282f9764b.

[66] Suzuki A, Brunt EM, Kleiner DE, Miquel R, Smyrk TC, Andrade RJ, et al. The use of liver biopsy evaluation in discrimination of idiopathic autoimmune hepatitis versus drug-induced liver injury. Hepatology 2011;54:931-999. doi:10.1002/hep.24481.

[67] Kleiner DE. The pathology of drug-induced liver injury. Semin Liver Dis 2009;29:364-372. doi:10.1055/s-0029-1240005.

[68] Korman JD, Volenberg I, Balko ], Webster ], Schiodt FV, Squires RH Jr, et al. Screening for Wilson disease in acute liver failure: a comparison of currently available diagnostic tests. Hepatology 2008;48:1167-1174. doi:10. 1002/hep.22446

[69] Raja K, Thung SN, Fiel MI, Chang C. Drug-induced steatohepatitis leading to cirrhosis: long-term toxicity of amiodarone use. Semin Liver Dis 2009;29: 423-428. doi:10.1055/s-0029-1240011.

[70] Vernier-Massouille G, Cosnes ], Lemann M, Marteau P, Reinisch W, Laharie $D$, et al. Nodular regenerative hyperplasia in patients with inflammatory bowel disease treated with azathioprine. Gut 2007;56:1404-1409. doi: 10.1136/gut.2006.114363.

[71] Ju HY, Jang JY, Jeong SW, Woo SA, Kong MG, Jang HY, et al. The clinical features of drug-induced liver injury observed through liver biopsy: focus on relevancy to autoimmune hepatitis. Clin Mol Hepatol 2012;18:213-218. doi: $10.3350 / \mathrm{cmh} .2012 .18 .2 .213$.

[72] Ramachandran R, Kakar S. Histological patterns in drug-induced liver disease. J Clin Pathol 2009;62:481-492. doi:10.1136/jcp.2008.058248.

[73] Kleiner DE, Chalasani NP, Lee WM, Fontana RJ, Bonkovsky HL, Watkins PB, et al. Hepatic histological findings in suspected drug-induced liver injury: systematic evaluation and clinical associations. Hepatology 2014;59: 661-670. doi:10.1002/hep.26709.

[74] Rutherford AE, Hynan LS, Borges CB, Forcione DG, Blackard JT, Lin W, et al. Serum apoptosis markers in acute liver failure: a pilot study. Clin Gastroenterol Hepatol 2007;5:1477-1483. doi:10.1016/j.cgh.2007.08.007.

[75] Bantel H, Lugering A, Heidemann J, Volkmann X, Poremba C, Strassburg CP, et al. Detection of apoptotic caspase activation in sera from patients with 
chronic HCV infection is associated with fibrotic liver injury. Hepatology 2004;40:1078-1087. doi:10.1002/hep.20411.

[76] Rutherford A, King LY, Hynan LS, Vedvyas C, Lin W, Lee WM, et al. Development of an accurate index for predicting outcomes of patients with acute liver failure. Gastroenterology 2012;143:1237-1243. doi:10.1053/j.gastro.2012.07.113.

[77] Wetmore BA, Brees DJ, Singh R, Watkins PB, Andersen ME, Loy J, et al. Quantitative analyses and transcriptomic profiling of circulating messenger RNAs as biomarkers of rat liver injury. Hepatology 2010;51:2127-2139. doi:10.1002/hep.23574.

[78] Antoine DJ, Dear JW, Lewis PS, Platt V, Coyle J, Masson M, et al. Mechanistic biomarkers provide early and sensitive detection of acetaminophen-induced acute liver injury at first presentation to hospital. Hepatology 2013;58: 777-787. doi:10.1002/hep.26294.

[79] Antoine DJ, Jenkins RE, Dear JW, Williams DP, McGill MR, Sharpe MR, et al. Molecular forms of HMGB1 and keratin-18 as mechanistic biomarkers for mode of cell death and prognosis during clinical acetaminophen hepatotoxicity. J Hepatol 2012;56:1070-1079. doi:10.1016/j.jhep.2011.12.019.

[80] Bell LN, Vuppalanchi R, Watkins PB, Bonkovsky HL, Serrano J, Fontana RJ, et al. Serum proteomic profiling in patients with drug-induced liver injury. Aliment Pharmacol Ther 2012;35:600-612.

[81] Maniratanachote R, Shibata A, Kaneko S, Yamamori I, Wakasugi T, Sawazaki $T$, et al. Detection of autoantibody to aldolase $B$ in sera from patients with troglitazone-induced liver dysfunction. Toxicology 2005;216:15-23. doi:10. 1016/j.tox.2005.07.012.

[82] Andersson U, Lindberg J, Wang S, Balasubramanian R, Marcusson-Stahl M, Hannula $M$, et al. A systems biology approach to understanding elevated serum alanine transaminase levels in a clinical trial with ximelagatran. Biomarkers 2009;14:572-586. doi:10.3109/13547500903261354.

[83] Shi Q, Hong H, Senior J, Tong W. Biomarkers for drug-induced liver injury. Expert Rev Gastroenterol Hepatol 2010;4:225-234. doi:10.1586/egh.10.8.

[84] Steuerwald NM, Foureau DM, Norton HJ, Zhou J, Parsons JC, Chalasani N, et al. Profiles of serum cytokines in acute drug-induced liver injury and their prognostic significance. PLoS One 2013;8:e81974. doi:10.1371/journal. pone.0081974.

[85] Hammerich L, Heymann F, Tacke F. Role of IL-17 and Th17 cells in liver diseases. Clin Dev Immunol 2011;2011:345803. doi:10.1155/2011/345803.

[86] Li ], Zhu X, Liu F, Cai P, Sanders C, Lee WM, et al. Cytokine and autoantibody patterns in acute liver failure. J Immunotoxicol 2010;7:157-164. doi:10. 3109/15476910903501748.

[87] Wang K, Zhang S, Marzolf B, Troisch P, Brightman A, Hu Z, et al. Circulating microRNAs, potential biomarkers for drug-induced liver injury. Proc Natl Acad Sci USA 2009;106:4402-4407. doi:10.1073/pnas.0813371106.

[88] McGill MR, Sharpe MR, Williams CD, Taha M, Curry SC, Jaeschke H. The mechanism underlying acetaminophen-induced hepatotoxicity in humans and mice involves mitochondrial damage and nuclear DNA fragmentation. J Clin Invest 2012;122:1574-1583. doi:10.1172/JCI59755.

[89] Bharadwaj M, Illing P, Theodossis A, Purcell AW, Rossjohn J, McCluskey J. Drug hypersensitivity and human leukocyte antigens of the major histocompatibility complex. Annu Rev Pharmacol Toxicol 2012;52:401-431. doi:10. 1146/annurev-pharmtox-010611-134701.

[90] McCormack M, Alfirevic A, Bourgeois S, Farrell J], Kasperaviciute D, Carrington $\mathrm{M}$, et al. HLA-A*3101 and carbamazepine-induced hypersensitivity reactions in Europeans. N Engl J Med 2011;364:1134-1143. doi:10. 1056/NEJMoa1013297.

[91] Lee WM. Drug-induced hepatotoxicity. N Engl J Med 1995;333:1118-1127. doi:10.1056/NEJM199510263331706.

[92] Reuben A. Hy's law. Hepatology 2004;39:574-578. doi:10.1002/hep. 20081.

[93] Bjornsson E, Olsson R. Outcome and prognostic markers in severe druginduced liver disease. Hepatology 2005;42:481-489. doi:10.1002/hep. 20800.

[94] Tan HH, Chang CY, Martin P. Acetaminophen hepatotoxicity: current management. Mt Sinai J Med 2009;76:75-83. doi:10.1002/msj.20065.

[95] Dertinger S, Dirschmid K, Vogel W, Drexel H. Immunosuppressive therapy for carbamazepine-induced hypersensitivity syndrome and hepatitis. J Hepatol 1998;28:356-357. doi:10.1016/0168-8278(88)80027-8.

[96] Bjornsson E, Talwalkar J, Treeprasertsuk S, Kamath PS, Takahashi N, Sanderson S, et al. Drug-induced autoimmune hepatitis: clinical characteristics and prognosis. Hepatology 2010;51:2040-2048. doi:10.1002/hep.23588.

[97] Rakela J, Mosley JW, Edwards VM, Govindarajan S, Alpert E. A doubleblinded, randomized trial of hydrocortisone in acute hepatic failure. The Acute Hepatic Failure Study Group. Dig Dis Sci 1991;36:1223-1228.

[98] Hou FQ, Zeng Z, Wang GQ. Hospital admissions for drug-induced liver injury: clinical features, therapy, and outcomes. Cell Biochem Biophys 2012;64:77-83. doi:10.1007/s12013-012-9373-y.
[99] Makin AJ, Wendon J, Williams R. A 7-year experience of severe acetaminophen-induced hepatotoxicity (1987-1993). Gastroenterology 1995;109: 1907-1916. doi:10.1016/0016-5085(95)90758-0.

[100] Gunay-Aygun M, Font-Montgomery E, Lukose L, Tuchman Gerstein M, Piwnica-Worms K, Choyke $\mathrm{P}$, et al. Characteristics of congenital hepatic fibrosis in a large cohort of patients with autosomal recessive polycystic kidney disease. Gastroenterology 2013;144:112-121.e2. doi:10.1053/j. gastro.2012.09.056.

[101] Lee WM, Hynan LS, Rossaro L, Fontana RJ, Stravitz RT, Larson AM, et al. Intravenous $\mathrm{N}$-acetylcysteine improves transplant-free survival in early stage non-acetaminophen acute liver failure. Gastroenterology 2009;137: 856-864.e1. doi:10.1053/j.gastro.2009.06.006.

[102] Redeker AG, Yamahiro HS. Controlled trial of exchange-transfusion therapy in fulminant hepatitis. Lancet 1973;1:3-6. doi:10.1016/S0140-6736(73) 91220-8.

[103] Akdogan M, Camci C, Gurakar A, Gilcher R, Alamian S, Wright H, et al. The effect of total plasma exchange on fulminant hepatic failure. J Clin Apher 2006;21:96-99.

[104] Kondrup J, Almdal T, Vilstrup H, Tygstrup N. High volume plasma exchange in fulminant hepatic failure. Int ] Artif Organs 1992;15:669-676.

[105] Lee WM. Drug-induced hepatotoxicity. N Engl ] Med 2003;349:474-485. doi:10.1056/NEJMra021844.

[106] Larsen FS, Hansen BA, Jorgensen LG, Secher NH, Kirkegaard P, Tygstrup N. High-volume plasmapheresis and acute liver transplantation in fulminant hepatic failure. Transplant Proc 1994;26:1788.

[107] Banares R, Nevens F, Larsen FS, Jalan R, Albillos A, Dollinger M, et al. Extracorporeal albumin dialysis with the molecular adsorbent recirculating system in acute-on-chronic liver failure: the RELIEF trial. Hepatology 2013; 57:1153-1162. doi:10.1002/hep.26185.

[108] Kribben A, Gerken G, Haag S, Herget-Rosenthal S, Treichel U, Betz C, et al. Effects of fractionated plasma separation and adsorption on survival in patients with acute-on-chronic liver failure. Gastroenterology 2012;142: 782-789.e3. doi:10.1053/j.gastro.2011.12.056.

[109] Pless G. Artificial and bioartificial liver support. Organogenesis 2007;3: 20-24.

[110] Spagnuolo MI, Iorio R, Vegnente A, Guarino A. Ursodeoxycholic acid for treatment of cholestasis in children on long-term total parenteral nutrition: a pilot study. Gastroenterology 1996;111:716-719. doi:10.1053/gast. 1996.v111.pm8780577.

[111] Oppert M, Rademacher S, Petrasch K, Jorres A. Extracorporeal liver support therapy with Prometheus in patients with liver failure in the intensive care unit. Ther Apher Dial 2009;13:426-430. doi:10.1007/s00063-013-0321-4.

[112] Fontana RJ, Watkins PB, Bonkovsky HL, Chalasani N, Davern T, Serrano J, et al. Drug-Induced Liver Injury Network (DILIN) prospective study: rationale, design and conduct. Drug Saf 2009;32:55-68. doi:10. 2165/00002018-200932010-00005.

[113] Andrade RJ, Lucena MI, Kaplowitz N, Garcia-Munoz B, Borraz Y, Pachkoria K, et al. Outcome of acute idiosyncratic drug-induced liver injury: Long-term follow-up in a hepatotoxicity registry. Hepatology 2006;44:1581-1588. doi:10.1002/hep.21424.

[114] Bjornsson E, Kalaitzakis E, Av Klinteberg V, Alem N, Olsson R. Long-term follow-up of patients with mild to moderate drug-induced liver injury. Aliment Pharmacol Ther 2007;26:79-85. doi:10.1111/j.1365-2036.2007. 03355.x.

[115] Ostapowicz G, Fontana RJ, Schiodt FV, Larson A, Davern TJ, Han SH, et al. Results of a prospective study of acute liver failure at 17 tertiary care centers in the United States. Ann Intern Med 2002;137:947-954.

[116] Bjornsson E, Nordlinder H, Olsson R. Clinical characteristics and prognostic markers in disulfiram-induced liver injury. J Hepatol 2006;44:791-797. doi: 10.1016/j.jhep.2005.12.016.

[117] Wei G, Bergquist A, Broome U, Lindgren S, Wallerstedt S, Almer S, et al. Acute liver failure in Sweden: etiology and outcome. J Intern Med 2007; 262:393-401. doi:10.1111/j.1365-2796.2007.01818.x.

[118] Abboud G, Kaplowitz N. Drug-induced liver injury. Drug Saf 2007;30:277294.

[119] Schmidt LE, Larsen FS. MELD score as a predictor of liver failure and death in patients with acetaminophen-induced liver injury. Hepatology 2007;45: 789-796. doi:10.1002/hep.21503.

[120] O'Grady JG, Alexander G], Hayllar KM, Williams R. Early indicators of prognosis in fulminant hepatic failure. Gastroenterology 1989;97:439-445.

[121] Yamagishi Y, Saito H, Ebinuma H, Kikuchi M, Ojiro K, Kanamori H, et al. A new prognostic formula for adult acute liver failure using computer tomography-derived hepatic volumetric analysis. J Gastroenterol 2009;44:615623. doi:10.1007/s00535-009-0045-7.

[122] Chalasani NP, Hayashi PH, Bonkovsky HL, Navarro VJ, Lee WM, Fontana RJ, et al. ACG Clinical Guideline: The Diagnosis and Management of Idiosyncratic Drug-Induced Liver Injury. Am J Gastroenterol 2014;109:950-966. doi: 10.1038/ajg.2014.131. 